\title{
CHEMICALLY PREPARED LEAD MAGNESIUM NIOBATE DIELECTRICS
}

B.A. TUTTLE, J.A. VOIGT, D.L. SIPOLA, W.R. OLSON and D.M. Goy Sandia National Laboratories, Albuquerque, NM 87185

\section{ABSTRACT}

RECEIVED

JAN 301998

A chemical solution powder synthesis technique has been developed that produce $\mathrm{AiR}, \mathrm{T}$ I uniform powders of lead magnesium niobate (PMN) with 60 to $80 \mathrm{~nm}$ crystallite size. The synthesis technique was based on the dissolution of lead acetate and alkoxide precursors in acetic acid followed by precipitation with oxalic acid/propanol solutions. Lead magnesium niobate ceramics fabricated from these chemically derived powders had smaller, more uniform grain size and higher dielectric constants than ceramics fabricated from mixed oxide powders that were processed under similar thermal conditions. Chem-prep PMN dielectrics with peak dielectric constants greater than 22,000 and polarizations in excess of $29 \mu \mathrm{C} / \mathrm{cm}^{2}$ were obtained for $1100^{\circ} \mathrm{C}$ firing treatments. Substantial decreases in dielectric constant and polarization were measured for chemically prepared PMN ceramics fired at lower temperatures, consistent with previous work on mixed oxide materials.

\section{INTRODUCTION}

Lead magnesium niobate (PMN) dielectrics are of great interest for high strain, low hysteresis microacuators, high dielectric constant capacitors and sensors. Greater mechanical strength, improved phase purity and more uniform grain size are desired for bulk actuator, micropositioner and thin multilayer capacitor applications. The decrease in strength of PMN mixed oxide ceramics compared to conventional lead zirconate titanate materials is in part due to the large, nonuniform grain size that accompanies the thermal treatments necessary to produce high dielectric constant, high electrostrictive coefficient PMN ceramics. Enhanced chemical homogeneity on the submicrometer scale via chemical preparation techniques is expected to lead to improved phase purity and more uniform grain size PMN ceramics. Specific issues associated with improvement of phase purity are the elimination of low dielectric constant pyrochlore phases and $\mathrm{PbO}$ rich grain boundary phases to produce high dielectric constant, single phase perovskite ceramics. For these reasons, we have investigated the chemical preparation of PMN powders. We have demonstrated that PMN ceramics with fine, uniform grain size and enhanced chemical homogeneity can be fabricated. These microstructural improvements should lead to better mechanical and electrical properties than those obtained by conventional mixed oxide processing.

The most widely accepted process for fabrication of mixed oxide PMN ceramics was developed by Swartz and coworkers. [1] A critical step in this process was the mixing and thermal reaction of $\mathrm{MgO}$ and $\mathrm{Nb}_{2} \mathrm{O}_{5}$ powder precursors to form powders of a magnesium/niobium solid solution columbite phase. Enhanced perovskite formation was obtained by combining these prereacted columbite powders with $\mathrm{PbO}$ powders, compared to mixing powders of individual components. Papet, Dougherty and Shrout [2] fabricated a series of PMN ceramics using fine, submicron, mixed oxide powders. Firing temperature was found to have a substantial effect on dielectric properties. Although substantial changes in grain size occurred for the different firing temperatures, a series dielectric mixing model was developed from TEM analyses, similar to the work of Wang and Schulze, [3] that indicated nanoscale dimension $(1 \mathrm{~nm}$ to $5 \mathrm{~nm})$ grain boundary phases were the primary cause for the dilution in dielectric constant with smaller grain size. Dielectric behavior of PMN ceramics with grain size below $1 \mu \mathrm{m}$ did not fit the series dielectric mixing model. Specifically, mixed oxide PMN dielectrics hot pressed at $810^{\circ} \mathrm{C}$ [2] developed a $0.3 \mu \mathrm{m}$ grain size and had a peak dielectric constant of 4600 , dielectrics conventionally fired at $1100^{\circ} \mathrm{C}$ possessed a $6.2 \mu \mathrm{m}$ grain size had a peak dielectric constant of 17,300 . These dielectric constants were measured at a frequency of $1 \mathrm{kHz}$. The peak dielectric constant of phase pure PMN for the series mixing model, dielectric constant - grain size relationships was on the order of 20,000 . A value similar to that obtained with chem-prep powders in this study.
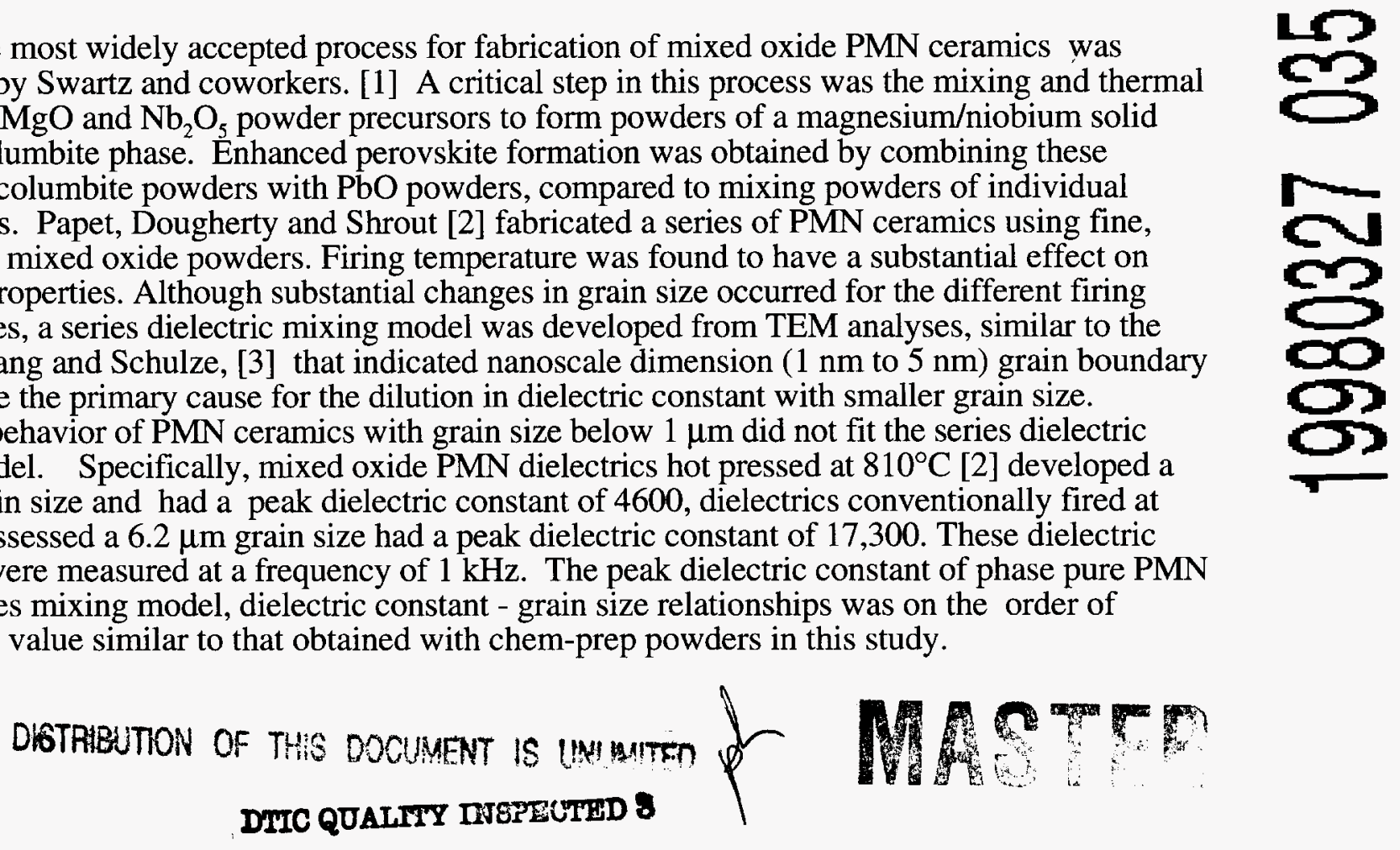


\section{DISCLAIMER}

This report was prepared as an account of work sponsored by an agency of the United States Government. Neither the United States Government nor any agency thereof, nor any of their employees, makes any warranty, express or implied, or assumes any legal liability or responsibility for the accuracy, completeness, or usefulness of any information, apparatus, product, or process disclosed, or represents that its use would not infringe privately owned rights. Reference herein to any specific commercial product, process, or service by trade name, trademark, manufacturer, or otherwise does not necessarily constitute or imply its endorsement, recommendation, or favoring by the United States Government or any agency thereof. The views and opinions of authors expressed herein do not necessarily state or reflect those of the United States Government or any agency thereof. 
A number of attempts have been previously made to synthesize chemically prepared PMN powders. Two different methodologies will be discussed. Chaput and coworkers [4] have fabricated fine powders from translucent gels of PMN using alkoxide precursors and hypercritical drying $\left(250^{\circ} \mathrm{C}\right.$ and $\left.6.3 \mathrm{MPa}\right)$. A B-site deficient pyrochlore phase was formed for temperatures below $700^{\circ} \mathrm{C}$, with a major percentage of perovskite phase being obtained by a $700^{\circ} \mathrm{C}$ thermal treatment. After an $800^{\circ} \mathrm{C}$ thermal treatment, the powders were single phase perovskite by $\mathrm{X}$-ray diffraction; however, no electrical results were given. Jeng and coworkers[5] have developed a process for PMN powder synthesis based on the coprecipitation of niobium oxalate, magnesium nitrate and lead nitrate. A $2 \mu \mathrm{m}$ to $4 \mu \mathrm{m}$ grain size material was developed at $900^{\circ} \mathrm{C}$, with a dielectric constant of 11,000 measured at ambient. The maximum dielectric constant measured in their study was 18,000 at $100 \mathrm{~Hz}$ at an unspecified lower temperature.

\section{EXPERIMENT}

Chem-prep PMN and PMN/PT 94/6 powders with 0.6 mol\% $\mathrm{Cu}$ addition were synthesized using an acetate / alkoxide / oxalic acid technique (Figure 1). [6] This powder synthesis technique minimizes waste and produces a readily filterable co-precipitate unlike many other chemical preparation techniques for lead based ferroelectrics.[7] In addition, a very uniform, weakly agglomerated, free flowing powder was obtained. While individual particles were on the order of $60 \mathrm{~nm}$, weak agglomerates of these fine particles in the form of $5 \mu \mathrm{m}$ to $10 \mu \mathrm{m}$ spheroids facilitated handling and green body formation. At least $99 \%$ of all of the original metal cation constituents were precipitated as determined by ICP analyses mass balance.

The metal cation containing solution was synthesized by first preparing a lead-magnesiumcopper acetate/acetic acid solution that was formed by dissolving lead oxide, magnesium acetate, and copper acetate in glatial acetic acid. This solution was mixed with a niobium-titanium butoxide/acetic acid solution to form the metal cation solution. An exothermic mixing reaction raised the solution temperature to about $40^{\circ} \mathrm{C}$. Moisture sensitivity and undesirable hydrolysis reactions were minimized by the use of titanium and niobium n-butoxides. The metal solution was then pumped into an oxalic acid / n-propanol precipitant solution to form a readily filterable oxalate coprecipitate. Advantages of the oxalic acid/n-proponal precipitant were the stoichiometric removal of constituent metal species and minimized agglomeration. While 1500 gram lots of PZT $95 / 5$ powder have been synthesized for other DOE programs using this process, 100 to 500 gram lots were synthesized for this development project.

A $400^{\circ} \mathrm{C}$ thermal treatment of the PMN powders to pyrolyze residual organics, followed by ball milling the pyrolyzed powders with $\mathrm{ZrO}_{2}$ media for 2 hours were critical process improvements that increased the densities of fired PMN materials compared to chem-prep PMN/PT 94/46 ceramics fabricated in the early stages of our study. Densities for PMN/PT ceramics fabricated without using these process steps were on the order of $90 \%$ of theoretical, while densities of PMN ceramics with the improved process were in excess of $96 \%$. While low field dielectric constants were $15 \%$ lower than mixed oxide materials for the unimproved process, the improved process resulted in dielectric constants that exceeded those of mixed oxide materials reported in the literature.[2]

Green body processing for the PMN based materials in this study, consisted of uniaxial pressing chem-prep PMN powder at $2 \mathrm{ksi}(13.7 \mathrm{MPa})$ and then isopressing the resultant compact at $30 \mathrm{ksi}(206 \mathrm{MPa})$. Green densities on the order of $59 \%$ were obtained. While no atmosphere powder was used for the $900^{\circ} \mathrm{C} / 1$ hour and $1100^{\circ} \mathrm{C} / 3$ hour PMN firings, a combination of bubbled alumina (hollow alumina spheres of approximately $2 \mathrm{~mm}$ diameter) and mixed oxide PMN/PT 94/6 powder was used to minimize weight loss during the $1000^{\circ} \mathrm{C} / 2$ hour firing. Typically, the weight loss was less than $1.3 \%$ for the PMN powders that contained 3 mol\% (1.90 weight \%) excess $\mathrm{Pb}$. 


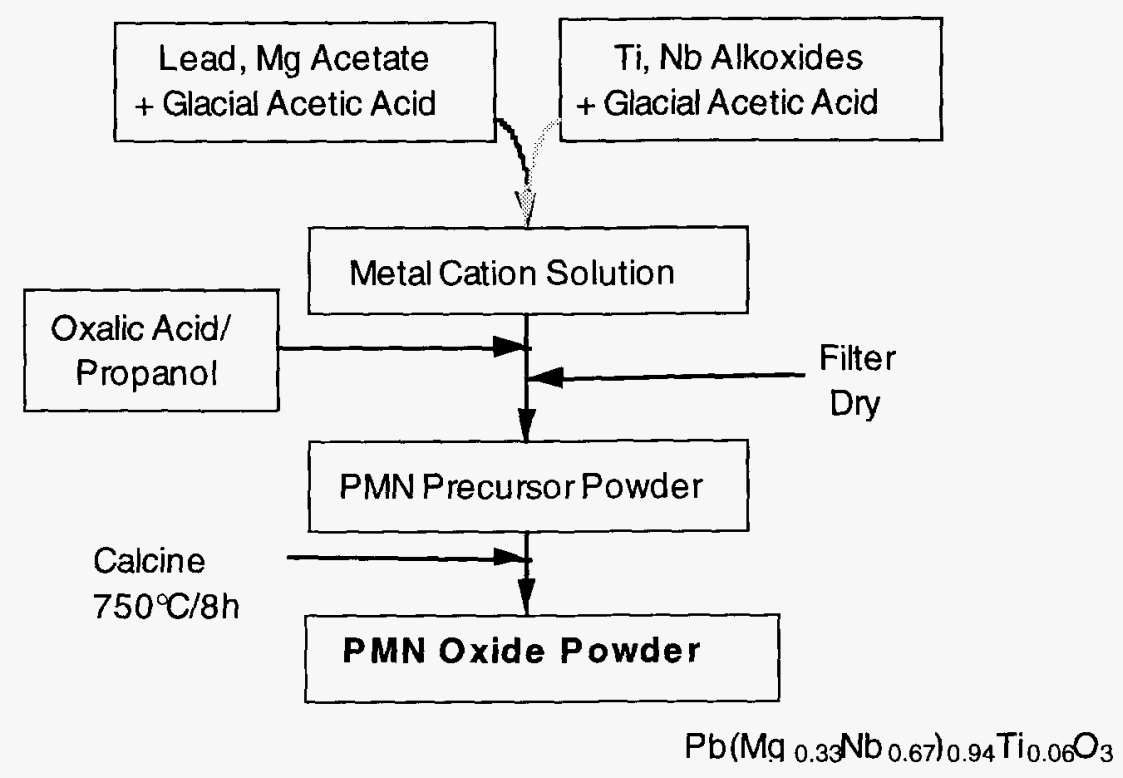

Figure 1. Flow Diagram for Chemical Preparation of Powders Technique for PMN/PT Ceramics.

Sample preparation for electrical measurements consisted of slicing $1 \mathrm{~mm}$ thick pellets from 15 gram, $2.22 \mathrm{~cm}$ diameter slugs. Defects in the pellet surfaces were reduced by lapping with 600 grit $\mathrm{SiC}$, followed by $9 \mu \mathrm{m}$ diamond and $1 \mu \mathrm{m}$ diamond abrasive polishes. The specimens were ultrasonically cleaned with acetone and then dried at $200^{\circ} \mathrm{C}$ for $30 \mathrm{~min}$ and cooled at $2{ }^{\circ} \mathrm{C} / \mathrm{min}$. $20 \mathrm{~nm} \mathrm{Cr} / / 100 \mathrm{~nm}$ Au electrodes were RF sputter deposited on the PMN materials. Dielectric constant versus temperature measurements were made on approximately $1 \mathrm{~mm}$ thick specimens with data being taken at $1^{\circ} \mathrm{C}$ intervals. An RT66A tester was used to measure polarization versus electric field characteristics at a frequency of approximately $2 \mathrm{~Hz}$. Sample preparation for microstructural analyses consisted of mounting specimens in epoxy, and polishing the PMN surface using a final $0.05 \mu \mathrm{m}$ colloidal silica polishing treatment. PMN samples were etched using a 600:11:1 solution of deionized water: $\mathrm{HF}: \mathrm{HCl}$ by weight [8]. While 2 min etch times were ideal for specimens with grain size greater than $4 \mu \mathrm{m}$, micrographs for samples with grain sizes less than $2 \mu \mathrm{m}$ were taken from regions of the sample that were only exposed to the etchant vapors.

\section{RESULTS}

\section{Density and Microstructure}

While a number of calcine temperature / time treatments were investigated, best results were obtained for a $750^{\circ} \mathrm{C} / 8 \mathrm{~h}$ calcination procedure. This treatment represented a compromise between having powder with high perovskite content and having fine particle powders with easily broken agglomerates. Roughly $30 \%$ pyrochlore phase was present by X-ray diffraction analyses. Pressed pellets fired at $1100^{\circ} \mathrm{C}$ were single phase perovskite by $\mathrm{X}$-ray diffraction analysis, but pellets fired at $1000^{\circ} \mathrm{C}$ and $900^{\circ} \mathrm{C}$ contained small amounts of litharge and pyrochlore. Improved calcine procedures for PMN powders have recently been developed by Narendar and Messing [9] by proper control of oxygen partial pressure and gas flow rates that results in phase pure materials for $750^{\circ} \mathrm{C}$ processing treatments. Adopting these calcination techniques for our powders should lead to enhanced perovskite phase purity at lower temperatures.

PMN density and weight loss for sintered PMN and PMN/PT ceramics that were fabricated for this study are shown in TABLE I. Densities for the chemically prepared PMN materials were 
comparable to commercial mixed oxide ceramics (TAM Ceramics 52639). Samples fabricated without the $400^{\circ} \mathrm{C}$ pyrolysis treatment followed by ball milling had densities that were approximately $6 \%$ to $8 \%$ lower than the commercial mixed oxide ceramics. No atmosphere powder was used for the firing treatments at $900^{\circ} \mathrm{C}$ and $1100^{\circ} \mathrm{C}$ shown in TABLE I. All chemically prepared ceramics had a nominal 3 mol\% (1.95 wt $\%$ ) excess $\mathrm{Pb}$ addition, while no $\mathrm{Pb}$ excess was assumed for the mixed oxide PMN/PT material. All weight losses during firing are attributed to the loss of $\mathrm{PbO}$, a standard assumption for $\mathrm{Pb}$ based dielectric processing. The weight losses given in TABLE I are with respect to the stoichiometric value of $\mathrm{PbO}$ for the composition. If the calculated value of $\mathrm{PbO}$ in the compact is in excess of the stoichiometric value, then this value is denoted by a parentheses. For example, for the chem-prep PMN $1100^{\circ} \mathrm{C}$ firing a weight loss compared to the $\mathrm{PbO}$ stoichiometric value of $1.15 \%$ was obtained, a $3.05 \%$ total weight loss, which corresponds to a weight loss of $3.05 \%$ of the fired sample compared to the bisque fired pellet. Because of the weight loss measured for the $1100^{\circ} \mathrm{C}$ firing, atmosphere powder consisting of $\mathrm{PMN}$ powder and bubbled alumina was used for the subsequent $1000^{\circ} \mathrm{C}$ firing. The fired weight of the $1000^{\circ} \mathrm{C}$ fired pellet was within $0.6 \%$ of the expected stoichiometric level. It should be noted that weight losses beyond that to maintain stoichiometry - similar to that observed for the $1100^{\circ} \mathrm{C}$ firing - may be advantageous to the removal of nanoscale dimension, low dielectric constant grain boundary phases. The presence of grain boundary phases can lower measured dielectric constants substantially. This dielectric constant dilution mechanism is covered in more detail in the excellent work by Pappet, Dougherty and Shrout [2].

TABLE I. Density and Weight Loss For PMN Dielectrics

\begin{tabular}{|l|c|c|c|}
\hline \multicolumn{1}{|c|}{ Composition } & Firing Treatment & Density (\% Theory) & Weight Loss (Gain) \\
\hline & & & $(1.2 \%)$ \\
\hline PMN & $900^{\circ} \mathrm{C} / 1$ hour & 89.9 & $(+0.6 \%)$ \\
\hline PMN & $1000^{\circ} \mathrm{C} / 2$ hours & 96.4 & $1.15 \%$ \\
\hline PMN & $1100^{\circ} \mathrm{C} / 3$ hours & 96.4 & $1.15 \%$ \\
\hline PMN/PT 94/6 & $1100^{\circ} \mathrm{C} / 1.5$ hours & 90.0 & $0.5 \%$ \\
\hline $\begin{array}{l}\text { PMN/PT 94/6 } \\
\text { Mixed Oxide }\end{array}$ & $1100^{\circ} \mathrm{C} / 3$ hours & 96.5 & \\
\hline
\end{tabular}

Microstructures of a chemically prepared PMN ceramic and a mixed oxide PMN/PT 94/6 ceramic are shown in Figure 2. Both materials were fired at $1100^{\circ} \mathrm{C}$. Previous work in our laboratory on mixed oxide PMN/PT ceramics showed a montonic trend of decreasing grain size with increasing $\mathrm{Ti}$ content, over the range of $0 \%$ to $100 \%$ Ti substitution. From this work, the slight difference in chemical composition between the two materials shown in Figure 2 has little effect on grain size. The chemically prepared material has substantially smaller grains (approximately $6.9 \mu \mathrm{m}$ grain size) than the mixed oxide material. Further the chem-prep PMN material is equiaxed, and has a relatively uniform grain size distribution. Grain size (GS) was determined using a lineal intercept technique with no multiplicative multiplier for three dimensional shape equivalence, as shown in equation 1 ,

$$
\mathrm{GS}=\mathrm{L} / \mathrm{N}^{*} \mathrm{M}
$$

where $\mathrm{L}$ is the total distance of measurement, $\mathrm{N}=$ the number of grain boundary intersections and $M$ is the magnification. The mixed oxide material shows very nonuniform grain growth, with grain sizes varying from $1 \mu \mathrm{m}$ to $34 \mu \mathrm{m}$. Smaller, uniform grain size should result in smaller, more uniform flaw sizes which can lead to higher mechanical strength and more robust dielectric breakdown behavior. The chem-prep microstrucure is also typified by dense grains with far fewer entrapped pores than the similarly processed mixed oxide material. 


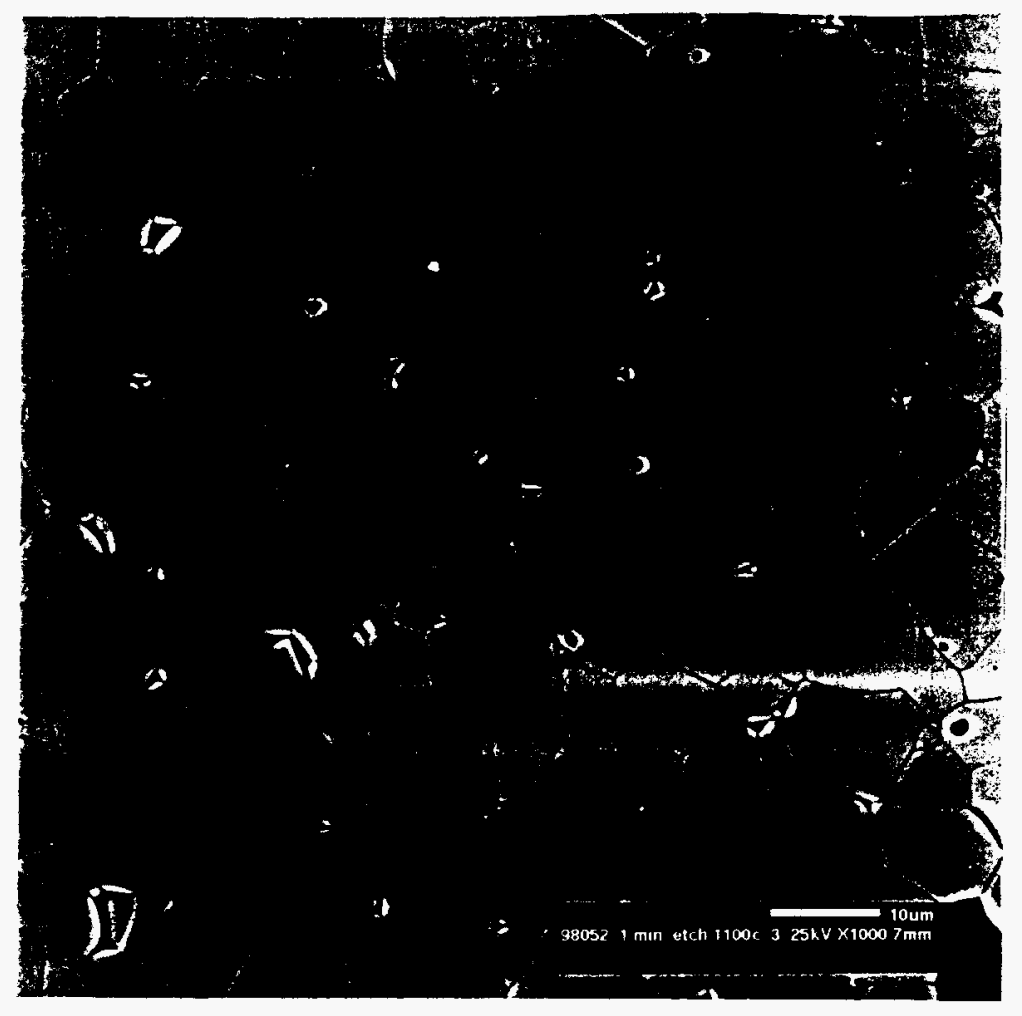

(A)

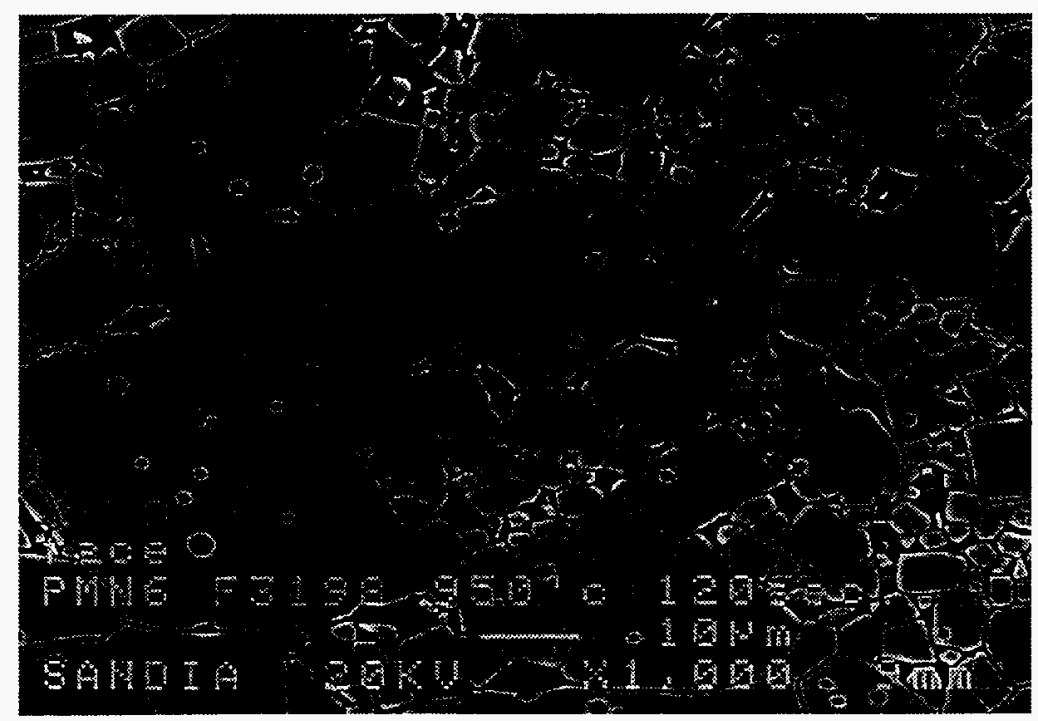

(B)

Figure 2. Microstructure of $\mathrm{PMN}$ based ceramics fired at $1100^{\circ} \mathrm{C}$ : A) chemically prepared PMN and B) mixed oxide PMN/PT 94/6. 
The evolution of microstructure of chemically prepared PMN materials is shown in Figure 3, for two chem-prep PMN ceramics fired at $900^{\circ} \mathrm{C}$ and $1000^{\circ} \mathrm{C}$, respectively. An extremely fine grain size is observed for the $900^{\circ} \mathrm{C}$ sample with a majority of grains being less than $0.1 \mu \mathrm{m}$. Some nonuniformity in growth is also evidenced, as clusters of grains on the order of $0.6 \mu \mathrm{m}$ are present. An average grain size of $0.3 \mu \mathrm{m}$ was measured using the lineal intercept technique. With increased firing temperature the microstructure coarsens, an average grain size of $0.82 \mu \mathrm{m}$ was measured for the chem-prep PMN sample fired at $1000^{\circ} \mathrm{C}$. However, the chem-prep PMN ceramics fired at this temperature still have extremely fine grain size compared to conventional mixed oxide materials. A bimodal distribution of grain size is observed for the chem-prep sample fired at $1000^{\circ} \mathrm{C}$ with the group of smaller grains being on the order of $0.3 \mu \mathrm{m}$ and the set of larger grains having an average grain size on the order of $1.0 \mu \mathrm{m}$. The larger grains may be attributed to micron scale regions of $\mathrm{Pb}$ enhancement. Polished sections of the chem-prep PMN powder after calcination at $750^{\circ} \mathrm{C}$ for 8 hours indicated $\mathrm{Pb}$ inhomgeneities on this scale. Nonetheless, these inhomgeneities in the chem-prep powder appear to be on a much finer scale than that observed for mixing mixed oxide powders with particle sizes on the order of several microns. Based on the improved dielectric constant results obtained for the chemically prepared materials, the chemical uniformity may also be better than that of submicron, mixed oxide powders.

\section{Dielectric Properties: Comparison of Chem-Prep and Mixed Oxide Ceramics}

Dielectric constants greater than 22,000 were obtained for chemically prepared PMN ceramics fired at $1100^{\circ} \mathrm{C}$, as shown in Figure 4. Conventional relaxor behavior, similar to that for mixed oxide materials, [2] is observed for the chem-prep PMN ceramics. As the measurement frequency increases, the magnitude of the peak dielectric constant, Kmax, decreases and the temperature at which the dielectric constant maximum occurs, Tmax, increases. This unusual dielectric relaxation behavior is the origin of the term relaxor ferroelectrics and is caused by the unique assemblages of polar nanoclusters within the material.[10] The values of Tmax for the frequencies of $100 \mathrm{~Hz}, 1 \mathrm{kHz}$, and $10 \mathrm{kHz}$ were $-7.9^{\circ} \mathrm{C},-5.5^{\circ} \mathrm{C}$ and $0^{\circ} \mathrm{C}$. These values agree with mixed oxide measurements [2] to within two degrees centigrade, which is within our experimental measurement error (TABLE II). The dielectric constant maxima are somewhat larger, on the order of $15 \%$, than the values previously measured for mixed oxide materials. Mixed oxide PMN data [2] is tabulated in the last row of TABLE II. Improved homogeneity of material, greater ordering of nanoclusters, and less nanodimension second phase at grain boundaries are among the factors that could be the cause of this property improvement.

TABLE II. Dielectric Transition Behavior for PMN Ceramics

\begin{tabular}{|c|c|c|c|c|c|}
\hline $\begin{array}{c}\text { Firing } \\
\text { Temperature }\end{array}$ & $\begin{array}{c}\text { Grain Size } \\
(\mu \mathrm{m})\end{array}$ & $\begin{array}{c}\mathrm{Kmax} \\
(100 \mathrm{~Hz})\end{array}$ & $\begin{array}{c}\text { Tmax } \\
(100 \mathrm{~Hz})\end{array}$ & $\begin{array}{c}\mathrm{Kmax} \\
(1 \mathrm{kHz})\end{array}$ & $\begin{array}{c}\text { Tmax } \\
(1 \mathrm{kHz})\end{array}$ \\
\hline $900^{\circ} \mathrm{C}$ & & & & & \\
\hline $1000^{\circ} \mathrm{C}$ & 0.3 & 2200 & $-6.0^{\circ} \mathrm{C}$ & 2150 & $-4.0^{\circ} \mathrm{C}$ \\
\hline $1100^{\circ} \mathrm{C}$ & 6.9 & 4000 & $-7.9^{\circ} \mathrm{C}$ & 3900 & $-4.9^{\circ} \mathrm{C}$ \\
\hline $\begin{array}{c}1100^{\circ} \mathrm{C} \\
\text { (Mixed Oxide) } \\
{[2]}\end{array}$ & 6.2 & 22,500 & $-9.9^{\circ} \mathrm{C}$ & 21,270 & $-5.9^{\circ} \mathrm{C}$ \\
\hline
\end{tabular}




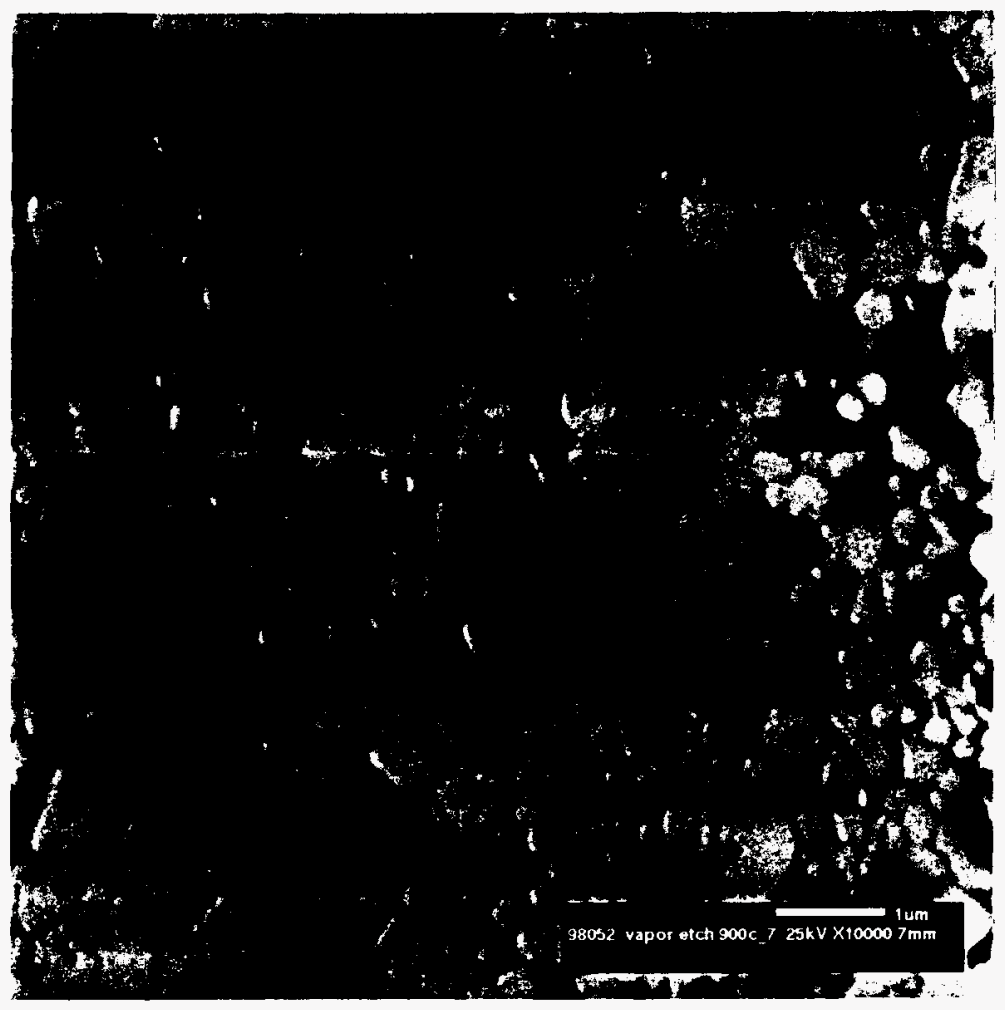

(A)

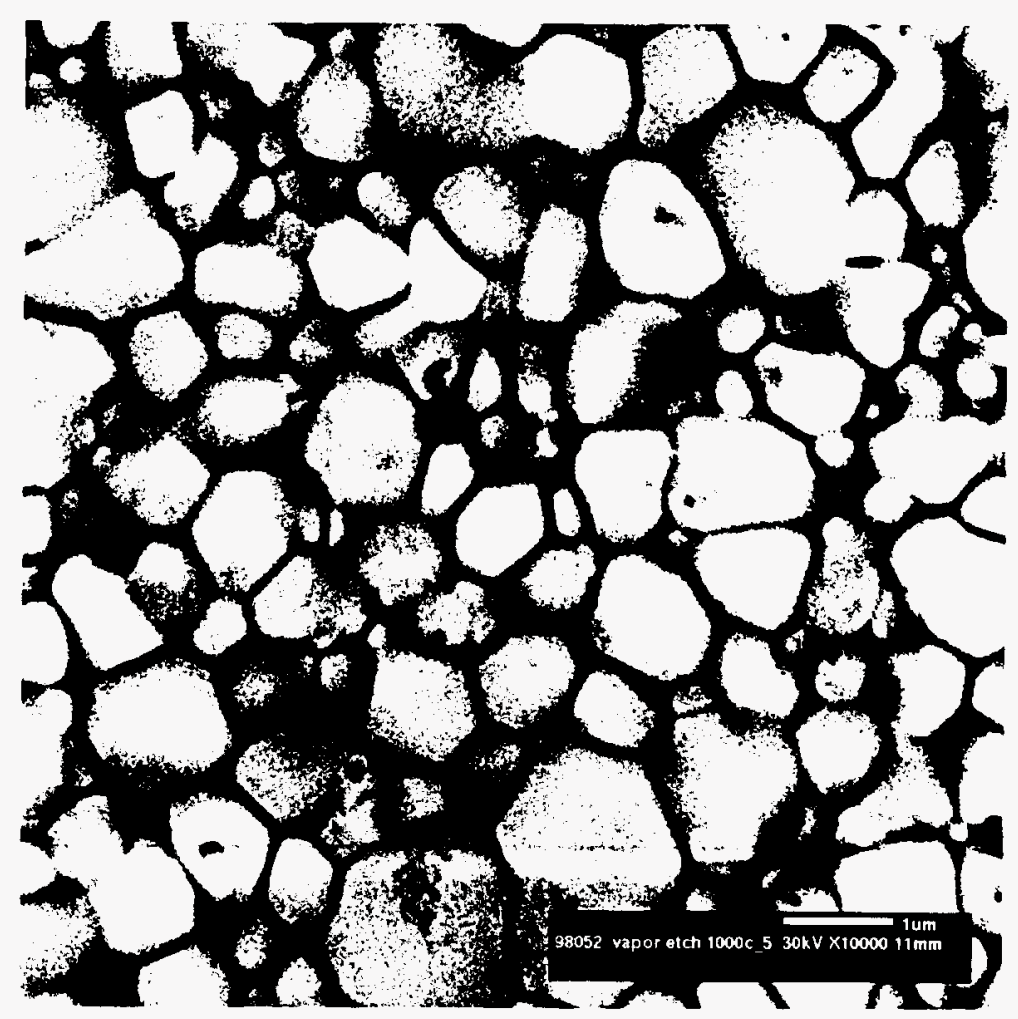

(B)

Figure 3. Microstructures of PMN ceramics fired at (A) $900^{\circ} \mathrm{C}$ and (B) $1000^{\circ} \mathrm{C}$. 


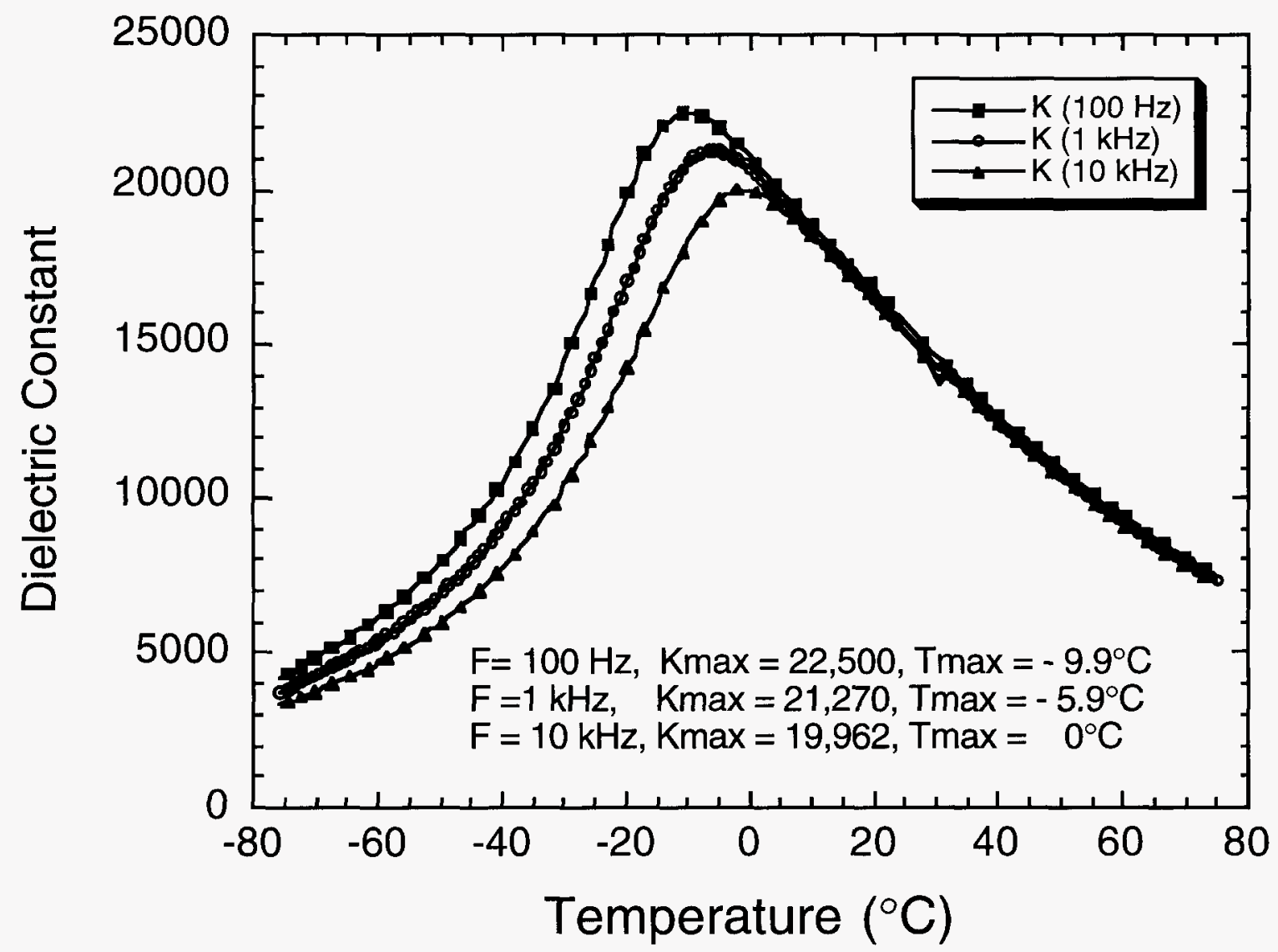

Figure 4. Dielectric constant versus temperature for chemically prepared PMN dielectrics fired at $1100^{\circ} \mathrm{C}$ for 3 hours.

Dielectric constants of the chemically prepared PMN ceramics in this study were a strong function of grain size, similar to that previously reported [2] for mixed oxide materials. For these PMN materials, the larger the grain size, the greater the dielectric constant. Peak dielectric constants of $21,270,3,900$ and 2500 were measured at $1 \mathrm{kHz}$ for chemically prepared PMN materials fired at $1100^{\circ} \mathrm{C}, 1000^{\circ} \mathrm{C}$ and $900^{\circ} \mathrm{C}$, respectively. The aforementioned firing treatments resulted in the following average lineal intercept grain sizes: $6.9 \mu \mathrm{m}, 0.82 \mu \mathrm{m}$ and $0.3 \mu \mathrm{m}$. Dielectric constant versus temperature characteristics for the chem-prep PMN ceramics fired at $1100^{\circ} \mathrm{C}$ and $1000^{\circ} \mathrm{C}$ are shown in Figure 5. The data is quantitatively quite similar to that of Pappet, Shrout, and Dougherty for mixed oxide PMN materials.[2] They measured peak dielectric constants of $18,000,11,000$ and 4,600 at $1 \mathrm{kHz}$, for PMN ceramics with grain sizes of $6.2 \mu \mathrm{m}, 1.1 \mu \mathrm{m}$ and $0.3 \mu \mathrm{m}$. While Pappet and coworkers attributed the dielectric constant dilution with grain size (down to $1 \mu \mathrm{m}$ ) to the presence of a grain boundary phase, other factors may affect our data. One factor is phase purity, evidence of second phases of both litharge and pyrochlore are detected in the X-ray patterns of the smaller grain size $900^{\circ} \mathrm{C}$ and $1000^{\circ} \mathrm{C}$ samples. Consideration of dielectric mixing rules would suggest that while the distribution of second phase pyrochlore would not be a major factor, a $\mathrm{PbO}$-rich grain boundary phase could lead to series dilution of the dielectric constant, as previously demonstrated. [2,3] Recent work by Samara [11] has demonstrated that relaxor behavior can be observed in some normal ferroelectrics by application of hydrostatic pressure. Further, as pressure is increased, the relaxor transition becomes more diffuse and the peak dielectric constant is lowered. Thus, the enhanced stress of fine grain, individual crystallites may reduce the peak of the dielectric constant for both mixed oxide and chem-prep materials. 


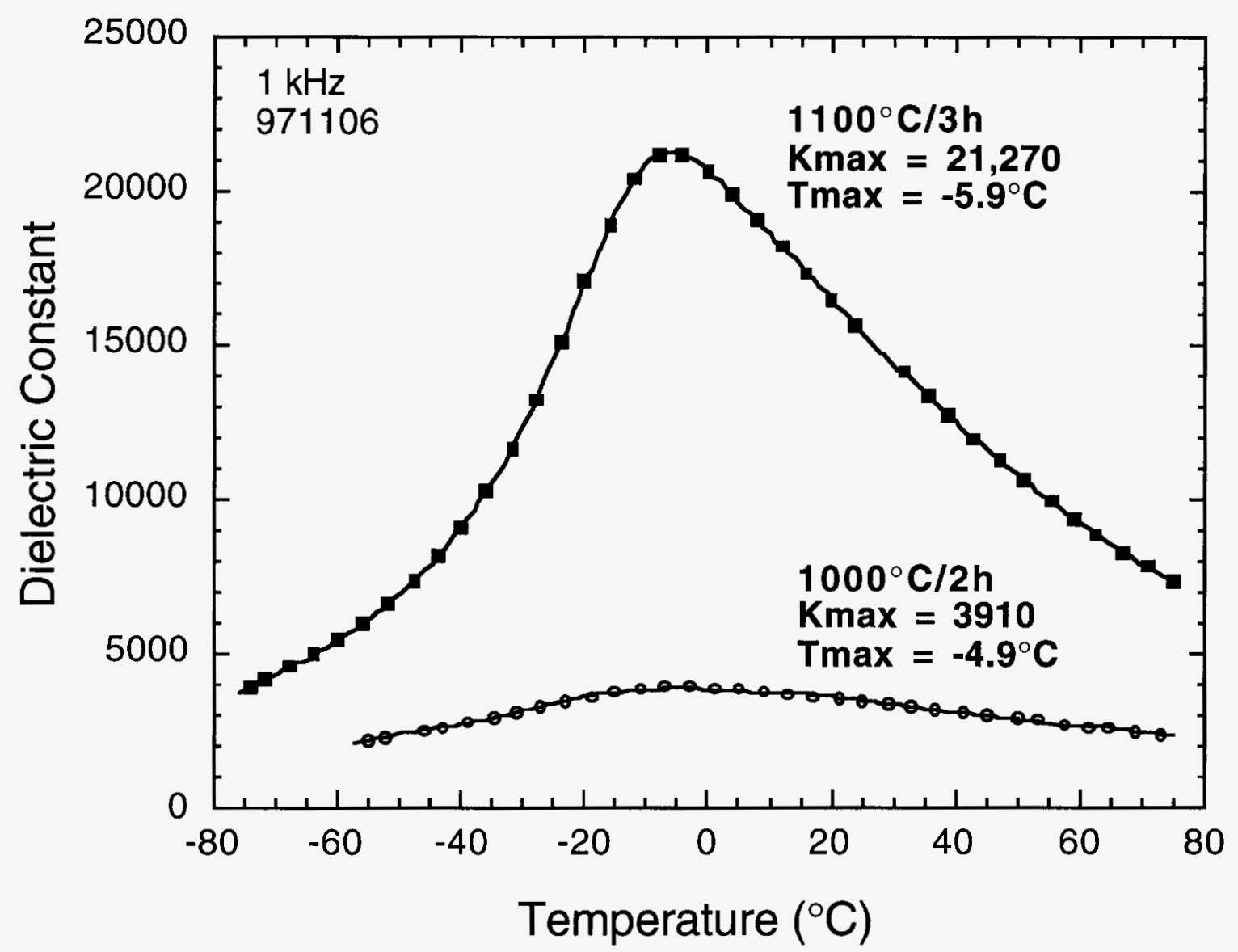

Figure 5. Dielectric Constant versus temperature for Chem-prep PMN ceramics fired at $1000^{\circ} \mathrm{C}$ and $1100^{\circ} \mathrm{C}$.

Polarization versus electric field behavior is shown in Figure 6 for the chemically prepared PMN ceramic fired at $1100^{\circ} \mathrm{C}$. An applied field of $30 \mathrm{kV} / \mathrm{cm}$ was used to obtain the polarization vs. field characteristic. The dielectric hysteresis characteristic is indicative of the slim loop, limited hysteresis, ferroelectric behavior common to relaxor materials. Maximum polarizations of 21, 25 and $29 \mu \mathrm{C} / \mathrm{cm}^{2}$ were obtained for field strengths of 20,30 and $40 \mathrm{kV} / \mathrm{cm}$, respectively. The samples fired at $900^{\circ} \mathrm{C}$ and $1000^{\circ} \mathrm{C}$ had substantially lower maximum polarizations than the larger grain size $1100^{\circ} \mathrm{C}$ sample. Maximum polarizations of $6.9 \mu \mathrm{C} / \mathrm{cm}^{2}$ and $11.4 \mu \mathrm{C} / \mathrm{cm}^{2}$ were obtained for applied fields of $40 \mathrm{kV} / \mathrm{cm}$ for the $900^{\circ} \mathrm{C}$ and $1000^{\circ} \mathrm{C}$ samples, respectively. Maximum polarizations of 5.4 and $9.4 \mu \mathrm{C} / \mathrm{cm}^{2}$ were obtained for applied fields of $30 \mathrm{kV} / \mathrm{cm}$ for the $900^{\circ} \mathrm{C}$ and $1000^{\circ} \mathrm{C}$ samples, respectively. Internal stress and the presence of a low dielectric constant second phase at the grain boundaries may be responsible for the substantial lower polarizations measured for the samples fired at $900^{\circ} \mathrm{C}$ and $1000^{\circ} \mathrm{C}$. 


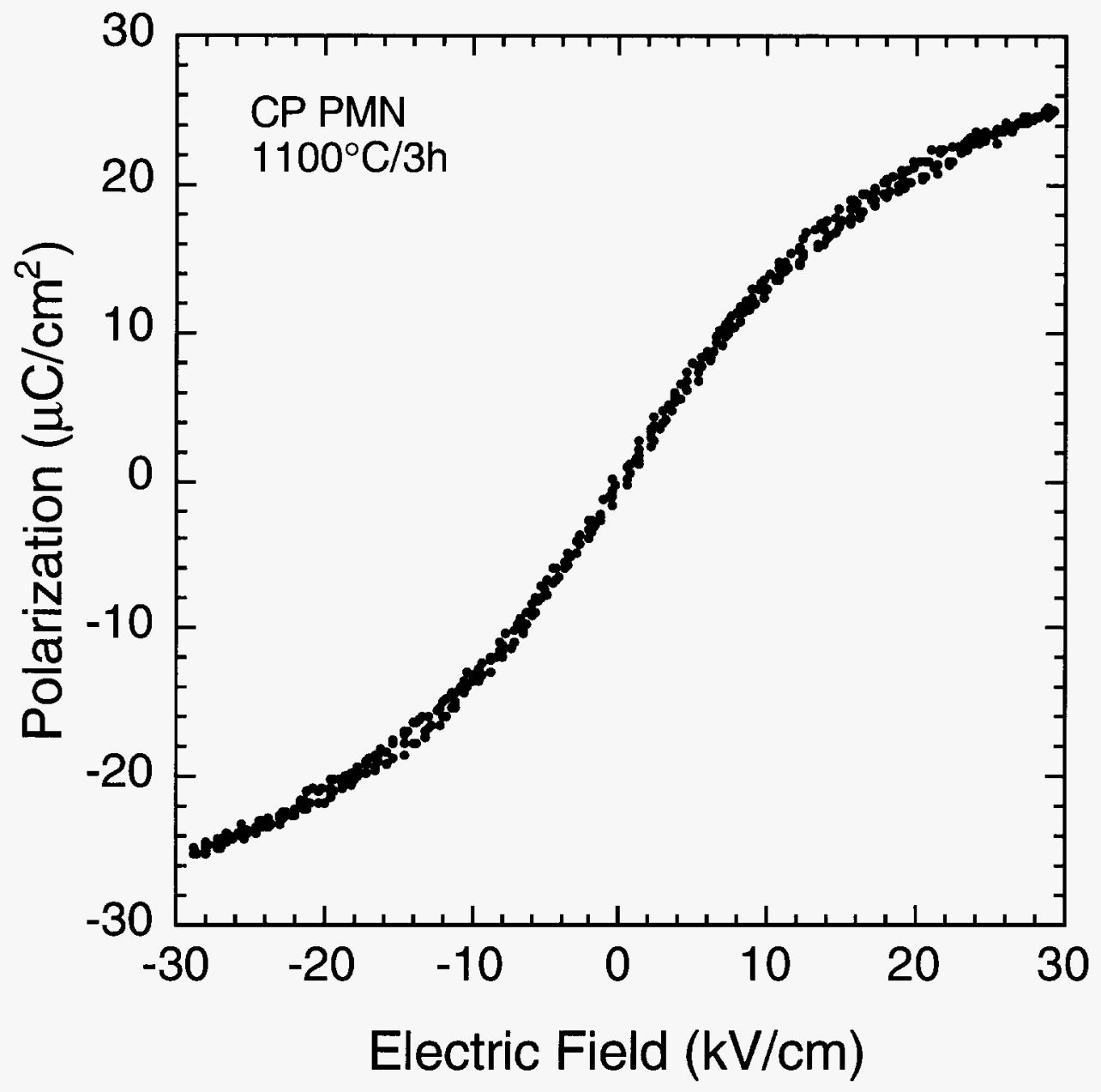

Figure 6. Polarization vs. Electric Field for Chemically Prepared PMN ceramic fired at $1100^{\circ} \mathrm{C}$ for 3 hours.

\section{SUMMARY}

A chemical powder synthesis technique was developed for PMN based powders using acetate / alkoxide precursors with an oxalic acid / propanol solution as the precipitating agent. Highly filterable powders were obtained from this nonaqueous process with minimal chemical waste. After calcination at $750^{\circ} \mathrm{C}$ for 8 hours, a particle size of approximately $60 \mathrm{~nm}$ and weak agglomerates of approximately $10 \mu \mathrm{m}$ dimension were obtained. PMN ceramics fabricated from these chemically derived powders had a finer, more uniform grain size than mixed oxide ceramics processed using similar thermal treatments. The more uniform, fine grain chem-prep PMN microstructure should be conducive to higher mechanical and dielectric breakdown strengths than those obtained from conventional mixed oxide PMN dielectrics. Peak dielectric constants $\left(\mathrm{K}_{\max }\right)$ in excess of 21,000 at $1 \mathrm{kHz}$ were obtained from the chemically prepared ceramics, compared to peak dielectric constants of 18,000 previously reported for highly reactive, mixed oxide powder PMN ceramics processed under similar conditions. 


\section{ACKNOWLEDGMENTS}

The authors acknowledge the fine technical efforts of Gary Zender and Alice Kilgo for SEM analysis and sample preparation. Further, technical discussions with Bob Brooks, Curtis King and Pat Smith with regard to electrical measurements and sample preparation were greatly appreciated. Sandia is a multiprogram laboratory operated by Sandia Corporation, a Lockheed Martin Company, for the United States Department of Energy under contract DE-ACO494AL85000.

\section{REFERENCES}

1. S. L. Swartz and T.R. Shrout, Mater. Res. Bull. XXVII, p.1245 (1982).

2. P. Papet, J.P. Dougherty, and T.R. Shrout, J. Mater. Res. 5, p.2902 (1990).

3. H.C. Wang and W.R. Schulze, J. Am. Cer. Soc. 73, p.825 (1990).

4. F. Chaput, J-P. Boilot, M. Lejeune, R. Papiernik, L.G. Huber-Pfalzgraf, J. Am. Ceram. Soc. 72, p. 1355 (1989).

5. D-Y. Jeng, C-J. Chen, S. R. Chaudhuri, Proceedings of the Eighth US-Japan Seminar on Dielectric and Piezoelectric Ceramics, p. 227 (1997).

6. J.A. Voigt, D.L. Sipola, B.A. Tuttle and M.T. Anderson, Sandia Technical Disclosure, SD6106 (1997).

7. G.H. Haertling and C.E. Land, Ferroelectrics, 3, p.269 (1972).

8. T. Hatanaka and H. Hasegawa, Jpn. J. Appl. Phys. 31 p.3245 (1992).

9. Y. Narendar and G.L. Messing, J. Amer. Ceram. Soc. 80, p.915 (1997).

10. L.E. Cross, Ferroelectrics 76, p.241 (1987).

11. G. A. Samara, Phys. Rev. 77, p.314 (1996). 
M98002580

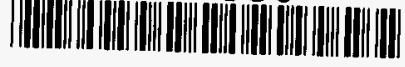

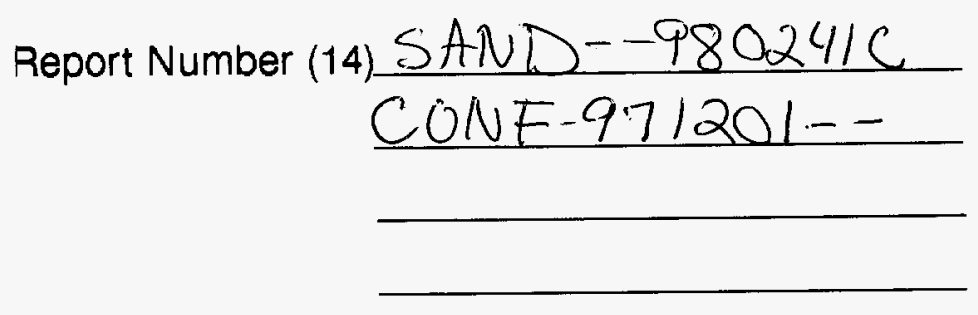

دubl. Date (11) 199801

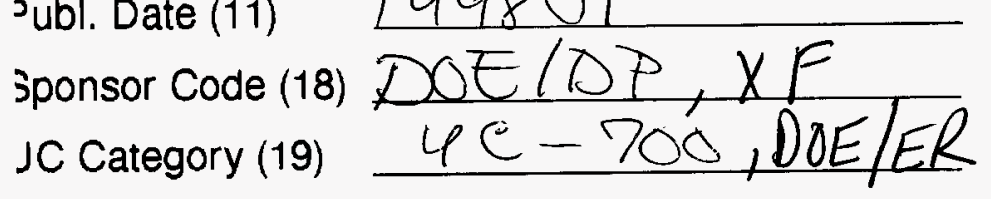

DOE 